\title{
The Knowledge Triangle in the Healthcare Sector - The Case of Three Medical Faculties in Norway
}

\author{
Siri Brorstad Borlaug \\ Senior Researcher, and Deputy Head of Research, siri.borlaug@nifu.no \\ Siri Aanstad \\ Researcher, siri.aanstad@nifu.no
}

Nordic Institute for Studies in Innovation, Research and Education (NIFU), PB 2815 Tøyen, 0608 Oslo, Norway

\begin{abstract}
$\mathrm{T}$

he paper investigates the social role of education and the relevance of university programs for meeting the real needs of society, which has gained particular political importance in recent years. Attention to this topic, in turn, has fueled interest in the concept of the «knowledge triangle», which implies a synergistic effect from the interplay of education, research, and innovation. Existing studies on the interaction of higher education institutions (HEIs) with society and policy in this field are primarily focused on the links between science and innovation and on the contributions of HEIs to economic development and growth. Many researchers focus on the interaction between universities and the industrial sector, but ignore HEIs' involvement in creating innovations in the public services sector. This is rather peculiar considering that innovation in the public sector has received increased policy attention over recent years and is seen as essential for improving the efficiency and quality of public services and for addressing

some of the major societal challenges, linked, for example, to an ageing population and maintaining the welfare state.

This paper looks at the healthcare sector, where HEIs interact with private industry as well as public healthcare services. It builds upon a study from Norway carried out in 2015 in the framework of an OECD project, which mapped and analyzed knowledge triangle policies and practices at the national and institutional level. This study showed that the interplay between education, research, and innovation is a key concern in national policy for the development of the healthcare sector and that knowledge triangle interactions with both the private and public sector is a central aspect of the current practices at medical departments at Norwegian HEIs. The linkages between the medical faculties and public healthcare services are especially interesting, as they provide patterns of interaction beyond those patterns identified in the existing literature and because education plays a central role.
\end{abstract}

Keywords: knowledge triangle; higher education; public sector; private sector; healthcare industry; Norway.

Article type: research paper

Citation: Brorstad Borlaug S., Aanstad S. (2018) The Knowledge Triangle in the Healthcare Sector - The Case of Three Medical Faculties in Norway. Foresight and STI Governance, vol. 12, no 1, pp. 80-87. DOI: $10.17323 / 2500-2597.2018 .1 .80 .87$

\footnotetext{
* For more details see the special issue of Foresight and STI Governance "Knowledge Triangle: Universities in Innovation System" (2017, vol. 11, no 2). - Editorial note.
} 
A s in policy, the focus of academic literature on the interaction between higher education institutions (HEIs) and society has primarily been on the relationship between research and innovation and the role university research may play in economic development and growth. Lately, the quest for HEIs that provide relevant education for society has entered the political agenda (e.g., [Norwegian Government, 2017]) and given rise to the re-introduction ${ }^{1}$ of the concept of the 'knowledge triangle', which assumes that there are potential synergies between education, research, and innovation.

Furthermore, reflecting the focus on the contribution of HEIs to economic growth, most studies have investigated their interaction with the industrial sector [Perkmann et al., 2013]. Little attention has - to our knowledge - been placed on how HEIs interact with and contribute to innovation in public sector services. This is rather peculiar considering that innovation in the public sector has received increased policy attention over recent years and is seen as essential for improving the efficiency and quality of public services and for addressing some of the major societal challenges that are linked, for example, to an aging population and maintaining a welfare state.

This paper looks at the healthcare sector, where HEIs interact with private industry as well as public healthcare services. It builds upon a study from Norway carried out in 2015 in the framework of an OECD project ${ }^{2}$, which mapped and analyzed knowledge triangle policies and practices on the national and institutional level [Borlaug et al., 2016]. The study shows that the interplay between education, research, and innovation is a key concern in national policies for the development of the healthcare sector, and that the interactions within the knowledge triangle with both the private and public sector are a critical aspect of current practices at the medical departments at Norwegian HEIs. The linkages between the medical departments and public healthcare services are especially interesting, as they provide other patterns of interaction beyond those identified in the existing literature and the fact that education plays a central role here.

\section{The Interaction between Higher Education Institutions and Society}

Many studies have investigated the relationship between research and innovation and the channels of interactions between HEIs and private firms. One strand of these studies focuses solely on 'entrepreneurial activities' building upon, amongst other things, insights from the studies of the entrepreneurial university (e.g., [Clark, 1998; Etzkowitz et al., 2000]). Entrepreneurial activities involve, on the one hand, entrepreneurial education programs and entrepreneurial research activities such as patenting, licensing, and start-ups as well as systemic and institutional initiatives for supporting and enhancing these types of activities with the use of technology transfer offices and science parks [Siegel et al., 2003; Clarysse et al., 2005; Perkmann et al., 2013]. This is a typical example of the knowledge triangle where student projects and the commercialization of research lead to the introduction of new products, processes, services, and businesses.

However, another strand of the literature has emphasized that the commercialization of research accounts for a relatively small part of the knowledge transfer from universities to society [Cohen et al., 2002; Schartinger et al., 2002; Bekkers, Bodas Freitas, 2008]. In fact, one study from Norway reports that no more than about six percent of the scientific staff engage in these types of activities [Thune et al., 2014]. Other and more important channels for interaction are collaborative and contract research [MeyerKrahmer, Schmoch, 1998; Perkman, Walsh, 2007; d'Este, Patel, 2007], mobility (university faculty working in industry/public sector and vice versa) [Gübeli, Doloreux, 2005; Bekkers, Bodas-Freitas, 2008], informal networks and conferences [Meyer-Krahmer, Schmoch, 1998; d'Este, Patel, 2007], and paid and unpaid consulting services [Amara et al., 2013]. These formal and informal channels enhance the potential for interlinkages between research and innovation as the HEI researchers obtain access to critical knowledge needs in the private sector while the private sector receives access to research at HEIs and may as such contribute both directly and indirectly to innovation.

As these studies show, we have relatively good insights into the linkages between HEIs and the private sector when it comes to research and innovation, but our knowledge about the channels of interaction with regard to education is more limited. There are, however, some relevant studies. Bekkers and BodasFreitas [Bekkers, Bodas-Freitas, 2008] have focused on the hiring of graduate students and student internship programs as important knowledge transfer channels, and Tømte et al. [Tømte et al., 2015] have emphasized continuing education. The latter study found that employees in both the public and private sector improve their knowledge bases and obtain access to relevant research through courses at HEIs, and that HEIs interact with employers to provide relevant courses. A survey of Norwegian academic staff shows that this was in fact one of the most important channels of interaction between HEIs and the public and private sectors [Thune et al., 2014]. The same survey also shows that academic staff more often collaborate with the public sector than the private sector, but these channels of interaction are, to

\footnotetext{
${ }^{1}$ Used in the Lisbon Strategy (2000-2010) [European Parliament, 2010], and under Sweden's EU Presidency in 2009.

${ }^{2}$ For more details see the special issue of Foresight and STI Governance "Knowledge Triangle: Universities in Innovation System" (2017, vol. 11, no 2). - Editor's note.
} 
our knowledge, poorly studied. There are many reasons for this: one - as pointed out above - is the emphasis on HEIs' role in economic development and growth. Another is that HEIs themselves in many countries belong to the public realm and have traditionally played a key role in educating public sector staff. Therefore, interactions with the public sector may be seen as an embedded part of HEIs' mandate. Finally, studies of innovation in the public sector seem to have focused on internal administrative, often technology-driven, processes, and not on cooperation with external actors [de Vries et al., 2016].

Against this background, it is especially interesting to investigate the channels of interaction between HEIs and public service providers, and how they collaborate on education, research, and innovation. The literature on the knowledge triangle concept assumes that the interaction between education, research, and innovation can be strengthened by so-called orchestration tools [Sjoer et al., 2016], which are the platforms and processes that may be found both at the systemic and institutional levels. In this paper, we focus on such tools and the many channels of interaction between three different medical departments and external actors in both the public and private sectors.

\section{The Norwegian System and Main Policies for Education, Research, and Innovation within the Healthcare Sector}

In Norway, state-owned universities and university colleges are mainly responsible for education and research within the medical sciences. Historically, there has been a division of labor between the different types of institutions. The universities have been responsible for the research-intensive scientific fields, such as medicine and dentistry, and the university colleges were responsible for shorter professional programs within nursing and other fields with relatively low levels of research. This picture is changing, however, as recent mergers between universities and university colleges have resulted in the establishment of integrated medical and healthcare faculties covering a broad range of different medical sciences.

Generally, the faculties of medicine and healthcare carry out education and research in close cooperation with the public healthcare system. The specialist healthcare services - or public hospitals - in Norway are organized as health trusts administered by regional healthcare authorities that are owned by the Ministry of Health and Care Services. The historic ties to the medical faculties at the universities have been very strong, and interactions between the public hospitals and the higher education sector have been institutionalized in various ways. First, the hospitals have a legal responsibility to take active part in the education of healthcare personnel, for example, by offering practical training to students at HEIs, which is a function for which they receive earmarked government funding. Research is also a legally stipulated task for the hospitals and the regional healthcare authorities receive dedicated research funding from the Ministry of Health and Care Services. The research funding is allocated to the hospitals that work in close cooperation with universities and university colleges. In accordance with government guidelines, the regional health authorities have cooperative bodies with HEIs in their respective regions that are responsible for the allocation of the research funding, as well as for the discussion of matters of mutual interest in the areas of education and research. Much of the research funding goes to projects involving both hospital and HEI staff, and collaboration between the professional and academic fields is moreover underpinned by the widespread use of dual affiliations. Collaboration between the university hospitals and medical faculties is particularly strong, with a high degree of integration in terms of staff, equipment, and infrastructure.

The primary healthcare system covers a broad range of services offered by the municipalities, which also cooperate with HEIs and, in particular, the institutions offering shorter healthcare education programs. However, the municipalities do not have the same explicit responsibility to carry out health-related education and research as the regional healthcare authorities, and therefore do not receive earmarked government funding for these tasks. This means that the cooperation between HEIs and the municipal healthcare services is not institutionalized in the same way because it primarily involves healthcare sciences and professions with limited research activity and relates mainly to education.

\section{Policies for Research and Innovation}

Over the past decade, several government ministries in Norway have initiated the so-called 21 Strategies, which are national research and innovation strategies within priority areas for research-based development and value creation in the $21^{\text {st }}$ century. There are currently nine such strategies for priority areas ranging from the oil and gas sector to health and care, which have been developed with the involvement of several ministries, research institutions, industry, and other societal stakeholders.

The Health\&Care21 strategy stands out by emphasizing the importance of an integrated approach to education, research, and innovation, and this policy explicitly refers to the knowledge triangle concept. Knowledge triangle interactions are seen as essential for the realization of the three main goals set out in the strategy, which are to achieve better public health, breakthrough research, industrial development, and economic growth.

The strategy is concerned with facilitating innovation through increased interactions between education, research, and the healthcare services, as well as between education, research, and industry. It recommends 
that many of the mechanisms that are in place to ensure cooperation between public hospitals and HEIs are introduced in the municipal healthcare services. This includes giving municipalities a stronger, more explicit legal responsibility to fulfil this task and dedicated funding for contributing to education and research, as well as the establishment of regional cooperative bodies for municipalities, HEIs, and other research institutions.

The linkages between educational and research institutions and industry are described as underdeveloped, reflecting - among other things - the limited size of the Norwegian healthcare industry and the lack of a culture of and incentives for cooperation. Thus, key recommendations include introducing incentives for HEIs and health trusts to engage in patenting, commercialization, and innovation cooperation with industry, as well as compulsory courses in entrepreneurship and innovation in healthcare-related educational programs.

Besides allocating research funding to the regional healthcare authorities, the Ministry of Health and Care allocates funding for healthcare-related research and innovation through the Research Council of Norway (RCN). Unlike research funding agencies in many other countries, the RCN covers all disciplines and research-performing sectors and provides support for industrial R\&D and research-based innovation. The Research Council has developed a separate policy for innovation in the public sector, where the fundamental idea is that interactions within the knowledge triangle should be strengthened through so-called practice-oriented R\&D. Practice-oriented R\&D takes place during the close cooperation between institutions for research and education and public sector professions, with the aim to develop research-based solutions to practical problems as well as to strengthen knowledge-based education and professional practice. The Research Council's efforts in this area have so far been targeting two sectors, the educational sector - spanning from kindergartens to higher and continuing education, and more recently, to the health, care, and welfare sector.

\section{Case Studies}

The case studies were performed as a part of the OECD study on the knowledge triangle [OECD, 2017], and are based on a predefined template. In order to ensure the variation and comparability of the sample, we studied three different medical faculties at three HEIs; the Faculty of Medicine at the Norwegian University of Science and Technology (NTNU), an integrated medical faculty at UiT - the Artic University of Norway, and The Faculty of Health at the University College Buskerud and Vestfold (HBV) ${ }^{3}$. The case descriptions below are based on document studies and interviews with the deans and a group interview with two to four members of the academic staff, all of which were conducted in 2015 (for more details see [Borlaug et al., 2016)]).

\section{NTNU Faculty of Medicine}

The Faculty of Medicine (FM) at NTNU is a classical medical faculty offering a medical doctorate program as well as bachelor's, master's, and $\mathrm{PhD}$ programs in several medical and healthcare-related areas, including a master's program in pharmacology. The faculty is organized in seven departments and hosts several research centers. ${ }^{4}$ The main areas of research include translational research, medical technology, health surveys, and biobanking.

As a medical faculty, the FM is strongly embedded in the regional healthcare services, with particularly close ties to the regional health authority Helse Midt-Norge and its subordinate hospitals. The faculty is fully integrated with St. Olav's Hospital, and the two institutions make up the Integrated University Hospital in Trondheim. The national system for cooperation between the specialist healthcare services and HEIs means that the FM has close institutionalized ties with Helse Midt-Norge. It is an important platform for interactions between education, research, and innovation. The integration of the FM and St. Olav's Hospital in the Integrated University Hospital is explicitly based on the idea of the knowledge triangle. In practical terms, the two institutions function as one organization, they are physically colocated and represented on each other's boards and have joint leadership meetings, cooperating bodies for education and research, and a high number of bridging positions.

The tight integration is also reflected in the funding sources of the faculty. Basic government funding accounted for $34 \%$ of total R\&D expenditures in 2013. Of this funding, $24 \%$ was Research Council funding, and $30 \%$ of the funding came from other public sources. The high share of funding from other public sources reflects the importance of research funding from Helse Midt-Norge, which makes up about $50 \%$ of external funding at the faculty. According to data of National R\&D statistics and NIFU, industry accounted for a small share of total R\&D expenditure in 2013 - less than $2 \%$.

Integrated education, research, and innovation cooperation with the specialist healthcare services, and particularly St. Olav's Hospital, is an essential part of the faculty's activity. Other types of cooperation such as with the primary healthcare services are considered to be important, but underdeveloped

\footnotetext{
${ }^{3}$ Abbreviations are built up from Norwegian-language titles of the HEIs. - Editorial note.

4 NTNU merged with three university colleges in 2016 and has now a different structure.
} 
because the majority of the educational programs are directed towards the specialist healthcare services. The faculty also has long traditions in close research and innovation collaboration with the technology departments at NTNU, for example, within the field of ultrasound technology, where it has resulted in a spin-off company which is now part of GE Vingmed Ultrasound. Still, there is potential for stronger cross-disciplinary cooperation, according to our respondents.

There has not been any systematic integration of innovation in the educational programs at the faculty, but the newly established master's program in pharmacology includes a mandatory course in innovation. The objective is to give the students an introduction to the drug development process "from idea to final product", and the course draws upon the expertise of the university's technology transfer office. Another initiative is earmarked funding for $\mathrm{PhD}$ positions in innovation projects. The faculty funded three $\mathrm{PhD}$ positions in innovation projects in 2014-2015, and another two positions in 2016.

Industry collaboration is widespread and takes many different forms. The FM has a cooperative agreement with GE Vingmed Ultrasound, and the company rents offices in the Integrated University Hospital, funds $\mathrm{PhD}$ and postdoctoral positions, and is involved in education and research at the faculty through parttime positions. Moreover, the faculty has hosted two Centres for Research-based Innovation in recent years, both with GE Vingmed Ultrasound as an industrial partner: Medical Imaging Laboratory, MI Lab (2007-2015), and the Centre for Innovative Ultrasound Solutions, CIUS, which was started up in 2015. CIUS is a collaboration with researchers from St. Olav's Hospital and technology departments at NTNU and around ten national and regional industrial partners. There are several master's students associated with the Centre, but our respondents point out that intellectual property rights issues prevent direct student involvement in research cooperation with the industrial partners.

Whereas cooperation with the specialist healthcare services is institutionalized, cooperation with industry and commercialization is largely dependent upon individual interest and drive, according to our respondents. For instance, one of our respondents established his own consultancy firm based on previous work experience in the medical industry. Another point they make is that education in many cases is the responsibility of the members of academic staff who are the least active as researchers, while those who engage in research and innovation may not take part in education, primarily because of time constraints. This may have a bearing on the interest in research and innovation among students, and good role models for knowledge triangle practice are considered important.

The faculty is also engaged in commercialization and makes active use of the university support system for innovation, including internal funding for the development of research ideas with innovative potential and the technology transfer office. One example of when researchers at NTNU and St. Olav's Hospital have collaborated closely with the TTO was the development of a method and surgical navigation device for the treatment of severe headaches, called MultiGuide.

\section{UiT - the Artic University of Norway - The Faculty of Health Sciences}

The Faculty of Health Sciences (FHS) covers the traditional academic areas of medicine, dentistry, pharmacology, and psychology, as well as the shorter professional programs such as nursing, physiotherapy, etc., which have traditionally been offered by the university colleges. FHS is strongly embedded in the public healthcare sector in Northern Norway and has close ties to the primary and specialist healthcare services and the dental care services in the region. Interactions with the public hospitals governed by the regional health authority Helse Nord are especially strong and there is a high degree of integration between the faculty and the University Hospital in Northern Norway (UNN), which is located on the university campus. Helse Nord is furthermore an important source of research funding for FHS. Local and regional industry plays a limited role as a collaborative partner and funding source and, with the exception of funding from Helse Nord, national research funding seems to be more important than regional funding.

The close ties between FHS and the healthcare services in northern Norway are reflected in the composition of the Faculty Board, where both UNN and a municipality in Troms County are represented. There are no industry representatives on the board. External representation is said to be important because it brings in stakeholder prospects and provides broader societal legitimacy for strategic decisions.

The national system for interaction between HEIs and specialist healthcare services provides an important platform for education, research, and innovation cooperation between the FHS and the public hospitals in northern Norway. The cooperative body with Helse Nord, which allocates the research funding that the regional health authority receives from the Ministry of Health and Care Services, is said to play a major role in developing cooperation channels between the faculty and the hospitals.

Cooperative bodies are in place at the level of individual hospitals as well and the FHS has worked systematically to develop an institutional basis for interactions with UNN. The two institutions have joint leadership meetings and joint education and research committees, which function as important arenas for regular strategic dialogue and joint initiatives.

There is furthermore extensive use of dual affiliations, through which hospital staff work at the FHS and academic staff work in the hospitals. FHS currently employs more than 300 people with their primary employment in specialist healthcare services, who are said to contribute significantly to the quality and 
relevance of the educational programs. Dual affiliations have traditionally been most common within medicine, but the FHS is working to increase the number across all healthcare sciences and professions. The faculty has, as the pioneering faculty in Norway and in cooperation with UNN, established 30 dual affiliations for both hospital and university staff within areas other than medicine. It is planned to expand this initiative to the municipal primary healthcare services. However, the municipalities' lack of tradition, an explicit mandate, and earmarked funding for active involvement in education and research poses a challenge both for the establishment of dual affiliations and for the systematic interactions between education, research, and professional practice in the primary healthcare services more generally.

The FHS has a strategic focus on innovation in education, and more specifically on developing new forms of education to meet the needs of the healthcare services. As an integrated healthcare faculty, the FHS places a strong emphasis on so-called "cross-professional learning" in the educational programs and has introduced joint courses for all students with the objective of teaching them how to interact and cooperate across healthcare professions. The faculty is also in the process of developing joint areas for practical training through various pilot projects carried out in close collaboration with the healthcare services. The projects have been initiated by dedicated faculty staff as well as by actors in the healthcare services, and embedded at the faculty level. This is seen as an example of innovation in education that has been directly motivated by the needs for new types of competence in the healthcare sector following a recent major national healthcare reform.

The FHS is also engaged in commercialization and innovative collaboration with industry, mainly in the areas of medical biology and pharmacology. It utilizes the services of the local technology transfer office and has collaborative projects with firms that include the Centre for Research-based Innovation, MabCent, marine bioactivities and drug discovery (2007-2015), and two industrial PhD projects at the Department of Pharmacology. Within the area of pharmacology, innovation is closely integrated in education at both bachelor's and master's levels, and the department is actively developing master's projects with direct industrial relevance.

\section{University College of Buskerud and Vestfold - The Faculty of Health Sciences}

The Faculty of Health Sciences (FHS) specializes in four areas of study - nursing, optometry, radiography, and health technology, as well as the promotion of healthcare. It offers shorter study programs that qualify students for healthcare professions within these areas, as well as programs and courses for specialization and further education by professional practitioners. These are areas with relatively weak research traditions, but their research activity and competence has been increased over time, and the faculty offers a cross-disciplinary $\mathrm{PhD}$ program in personalized healthcare (focused on the development of healthcare services based on practical needs).

The FHS engages in close cooperation with the local and regional healthcare sector, primarily the municipal primary healthcare services when it comes to education, both through practical training for students and continuing education for professional practitioners. Practical training is an important mechanism for the systematic interaction and knowledge exchange between the faculty and the healthcare services and contributes to the quality and relevance of education as well as continuous and incremental improvements in professional practice. Continued education also plays a central role in the development of healthcare services, and the FHS has an extensive portfolio of courses commissioned by actors in industry and working life, which are tailored to their particular needs.

Innovation in education is a central area of activity that includes the development of innovative educational designs, as well as teaching students about innovation. The faculty has, for example, worked systematically to integrate the innovation concept and innovative thinking in all bachelor's-level programs through a project with funding from the government's "Entrepreneurship in Education" initiative.

The way our respondents see it, knowledge triangle interactions are an inherent part of the activities of a medical faculty offering professional education in close cooperation with the healthcare services. A key point in this context is that innovation is understood broadly, as something that includes incremental improvements in healthcare services based on the continuous exchange of knowledge between students, academic staff, and healthcare professionals.

It is important to note that research at FHS is practice-oriented, illustrated by the fact that the faculty has received project funding from the Research Council's program for practice-oriented R\&D in health and welfare services. The projects link research, education, and professional practice, with the aim to strengthen the knowledge base and thereby improve the quality of the healthcare professional education and the healthcare services.

The main campus for the Faculty of Health is part of the Papirbredden Knowledge Park, where the university college is co-located with knowledge-based companies, innovation support agencies, and the regional innovation company Papirbredden Innovation, which was established with HBV as one of the founding partners and owners. The company is a collaboration with municipalities, private industry, and a national agency, furthermore it engages in innovation projects, commercialization, and business development within the region's priority areas. Papirbredden Innovation has health and welfare 
technology as a priority area. The university college is also represented on the board of the Driv Incubator, an Industrial Development Corporation of Norway (SIVA) incubator which specializes in healthcarerelated commercialization and start-ups. In close cooperation with Papirbredden Innovation, the faculty initiated a process in 2007 to establish a cluster of local technology firms specializing in the development of healthcare and welfare technology, primarily for the municipal primary healthcare services. The cluster, which has received funding from a public program, is an important platform for enhancing the systematic innovative collaboration between the FHS, municipalities, and private industry. The role the FHS plays in innovative projects is primarily that of a facilitator of innovation processes in the healthcare services, for example, it could do so through a scientific consultancy, competence development, and formative research. The commercialization of research results is not a central activity at the faculty.

In 2012, FHS opened a center for the testing and demonstration of the technology developed by the healthcare innovative cluster. The center brings together students and staff at the faculty, the technology firms, and municipalities as well as other users of health and welfare technology. The FHS uses the center actively for educational purposes, and the students are introduced to the new technologies through simulation training and lectures from technology producers and from users in the municipal healthcare services.

\section{Discussion and Conclusions}

As the case studies show, the differences in academic profiles between the three health faculties have a strong bearing on the intensity of the collaboration and the channels of interactions with the public and private sectors. Within research-intensive fields such as medicine and pharmacology, we find patterns: i) an entrepreneurial university where staff and students are involved in entrepreneurship and commercialization ii) institutionalized collaboration on education, research, and innovation with public hospitals; and iii) research-based innovation collaboration with private industry. Other health sciences with weaker research traditions are characterized by different patterns and notably collaborate with i) both public hospitals and municipal healthcare providers on education and incremental service innovation, and ii) with municipalities and private technology firms on the development and implementation of health and welfare technologies.

In particular, the faculties at NTNU and UiT that cover research-intensive medical sciences are involved in Centres for Research-based Innovation, as well as commercialization and entrepreneurial activities. Here we see patterns of what we can call the entrepreneurial knowledge triangle (i.e. [Clark, 1998]). NTNU and UiT also have strong integrated education, research, and innovation cooperation with the specialist healthcare services, and especially the university hospitals in their respective regions. These practices are intrinsically linked to the national system for interaction between the public hospitals and medical faculties, where the hospitals have a legal responsibility and receive dedicated government funding for engaging in education and research. There are strategic collaborative bodies in place, based on government regulations, which discuss matters of mutual interest in the areas of research and education and are responsible for the allocation of research funding. The system is also characterized by the extensive use of dual affiliations and the close physical integration between the medical faculties and the university hospitals. These top-down, formal, and institutionalized channels of interactions strengthen the opportunities for knowledge triangle practices between the specialist healthcare services and medical sciences.

The two faculties that offer shorter programs for professional healthcare education, UiT and HBV, cooperate with the municipal primary healthcare services when it comes to practical training for students and continuing education for healthcare professionals. This contributes to the development of competences based on the needs of the healthcare services and thereby improvements in professional practice. However, for several reasons there are fewer systematic and integrated knowledge triangle interactions than between the medical sciences and the specialist healthcare services. First, the primary healthcare services do not have the same explicit mandate to contribute to the education of healthcare personnel and do not receive government funding for engaging in practical training of students. Second, the shorter healthcare educational programs and the corresponding fields of professional practice have traditionally not been based on research to any significant extent. Thus, the collaboration concerns primarily the training of undergraduate students and the development of courses for continuing education and, to a lesser extent, research collaboration. However, the health faculty at HBV engages in extensive innovative collaboration with technology firms and municipalities on developing and implementing health and welfare technologies in the primary healthcare services.

The differences in collaboration patterns and the degree of institutionalized ties between the medical faculties and the hospitals, municipalities, and private firms illustrate the importance of long-term agreements and funding for collaboration on education, research, and innovation. Collaboration between HEIs and the private sector is primarily based on bottom-up initiatives, and it might be that good examples and practices of collaboration between HEIs and the public sector may be transferred to private sector collaboration. This implies, however, new types of policies at both the national and institutional levels. One way that HEIs may strengthen their knowledge triangle interactions with industry can be 
through establishing strategic long-term partnerships on research and collaboration with important firms. This may not only encompass one-on-one partnerships, but could involve multiple firms from the same sector. At the national level, there are already cluster programs that serve a similar function as they offer long-term funding for collaboration on education, research, and innovation. These may, however, be developed to include other instruments for collaboration such as the extended use of dual affiliations that emphasize other qualifications beyond research and scientific publications.

\section{References}

Amara N., Landry R., Halilem N. (2013) Faculty consulting in natural sciences and engineering: Between formal and informal knowledge transfer. Higher Education, vol. 65, no 3, pp. 359-384.

Bekkers R., Bodas-Freitas I.M. (2008) Analysing knowledge transfer channels between universities and industry: To what degree do sectors also matter? Research Policy, vol. 37, no 10, pp. 1837-1853.

Borlaug S.B., Aanstad S., Solberg E., Thune T.M. (2016) The knowledge triangle in policy and institutional practices - The case of Norway (NIFU Report no 66), Oslo: Nordic Institute for Studies in Innovation, Research and Education (NIFU).

Clark B.R. (1998) Creating entrepreneurial universities: Organizational pathways of transformation. Issues in Higher Education, New York: Elsevier.

Clarysse B., Wright M., Lockett A., van de Velde E., Vohora A. (2005) Spinning out new ventures: A typology of incubation strategies from European research institutions. Journal of Business Venturing, vol. 20, no 2, pp. 183-216.

Cohen W.M., Nelson R.R., Walsh J.P. (2002) Links and Impacts: The Influence of Public Research on Industrial R\&D. Management Science, vol. 48, no 1, pp. 1-23.

D'Este P., Patel P. (2007) University-industry linkages in the UK: What are the factors underlying the variety of interactions with industry? Research Policy, vol. 36, no 9, pp. 1295-1313.

De Vries H., Bekkers V., Tummers L. (2016) Innovation in the public sector: A systematic review and future research agenda. Public Administration, vol. 94, no 1, pp. 146-166.

Etzkowitz H., Webster A., Gebhardt C., Terra B.R.C. (2000) The future of the university and the university of the future: Evolution of ivory tower to entrepreneurial paradigm. Research Policy, vol. 29, no 2, pp. 313-330.

European Parliament (2010) The Lisbon Strategy 2000-2010: An analysis and evaluation of the methods used and results achieved, Brussels: European Parliament.

Gübeli M.H., Doloreux D. (2005) An empirical study of university spin-off development. European Journal of Innovation Management, vol. 8, no 3, pp. 269-282.

Meyer-Krahmer F., Schmoch U. (1998) Science-based technologies: University-industry interactions in four fields. Research Policy, vol. 27, no 8, pp. 835-851.

Norwegian Government (2017) Meld. St. 16 (2016-2017). Quality Culture in Higher Education. Report to the Storting (white paper), Oslo: Ministry of Education and Research. Available at: https://www.regjeringen.no/contentassets/ aee30e4b7d3241d5bd89db69fe38f7ba/en-gb/pdfs/stm201620170016000engpdfs.pdf, accessed 16.12.2017.

OECD (2017) Knowledge Triangle Synthesis Report. Enhancing the Contributions of Higher Education and Research to Innovation, Paris: OECD.

Perkmann M., Walsh K. (2007) University-industry relationships and open innovation: Towards a research agenda. International Journal of Management Reviews, vol. 9, no 4, pp. 259-280.

Perkmann M., Tartari V., McKelvey M., Autio E., Broström A., D’Este P., Fini R., Geuna A., Grimaldi R., Hughes A., Krabel S., Kitson M., Llerena P., Lissoni F., Salter A., Sobrero M. (2013) Academic engagement and commercialisation: A review of the literature on university-industry relations. Research Policy, vol. 42, no 2, pp. 423-442.

Schartinger D., Rammer C., Fischer M.M., Fröhlich J. (2002) Knowledge interactions between universities and industry in Austria: Sectoral patterns and determinants. Research Policy, vol. 31, no 3, pp. 303-328.

Siegel D.S., Waldman D., Link A. (2003) Assessing the impact of organizational practices on the relative productivity of university technology transfer offices: An exploratory study. Research Policy, vol. 32, no 1, pp. 27-48.

Sjoer E., Nørgaard B., Goossens M. (2016) From concept to reality in implementing the Knowledge Triangle. European Journal of Engineering Education, vol. 41, no 3, pp. 353-368.

Thune T.M., Aamodt P.O., Gulbrandsen M. (2014) Noder i kunnskapsnettverket: Forskning, kunnskapsoverføring og eksternt samarbeid blant vitenskapelig ansatte i UH-sektoren (NIFU Report 23/2014), Oslo: Nordic Institute for Studies in Innovation, Research and Education (NIFU).

Tømte C.E., Olsen D.S., Waagene E., Solberg E., Børing P., Borlaug S.B. (2015) Kartlegging av etter- og videreutdanningstilbud $i$ Norge (NIFU Report 39/2015), Oslo: Nordic Institute for Studies in Innovation, Research and Education (NIFU). 